\title{
Constraining the Properties of SNe Ia Progenitors from Light Curves
}

\author{
B. Sadler ${ }^{1}$, Peter Hoeflich ${ }^{1}$, E. Baron ${ }^{2}$, K. Krisciunas ${ }^{3}$, G. Folatelli ${ }^{4}$, \\ M. Hamuy ${ }^{4}$, M. Khokhlov ${ }^{5}$, M. Phillips ${ }^{6}$, N. Suntzeff ${ }^{3}$, and L. Wang \\ ${ }^{1}$ Dept. of Physics, Florida State University,USA \\ ${ }^{2}$ Dept. of Physics and Astronomy, University of Oklahoma, \\ ${ }^{3}$ Dept. of Physics, Texas A \& M University, \\ ${ }^{4}$ Dept. de Astronomia, Universidad de Chile, Santiago, Chile, \\ ${ }^{5}$ Dept. Astronomy and Astrophysics, University of Chicago, USA, \\ ${ }^{6}$ Las Campanas Observatory, La Serena, Chile
}

\begin{abstract}
We present an analysis of high precision V light curves (LC) for 18 local Type Ia supernovae (SNe Ia) as obtained with the same telescope and setup at the Las Campanas Observatory (LCO). This homogeneity provides an intrinsic accuracy of a few hundredths of a magnitude with respect to individual LCs and between different objects. Based on the single degenerate (SD) scenario, we identify patterns which have been predicted by model calculations as signatures of the progenitor and accretion rate which change the explosion energy and the amount of electron capture, respectively. Using these templates as principle components and the overdetermined system of SNe pairs, we reconstruct the properties of progenitors and progenitor systems. All LCO SNe Ia follow the brightness decline relation except 2001ay. After subtraction of the two components, the remaining scatter is reduced to $\approx 0.01^{m}-0.03^{m}$. SNe Ia seem to originate from progenitors with main-sequence masses $M_{M S}>3 M_{\odot}$ with the exception of two subluminous SNe Ia with $M_{M S}<2 M_{\odot}$. The component analysis indicates a wide range of accretion rates in the progenitor systems closing the gap to accretion induced collapses (AIC). SN1991t-like objects show differences in decline rate $(d m 15)$ but no tracers of our secondary parameters. This may point to a different origin such as the double degenerate or pulsating delayed detonation scenarios. SN2001ay does not follow the decline relation. It can be understood in the framework of C-rich white dwarfs (WDs), and this group may produce an anti-Phillips relation. We suggest that this may be a result of a common envelope phase and mixing during central He burning as in SN1987A.
\end{abstract}

Keywords. stars: white dwarfs, supernovae - nuclear reactions, nucleosynthesis, abundances - methods: data analysis

\section{Introduction}

SNe Ia are thermonuclear explosions of WDs (Hoyle \& Fowler 1960), i.e. end-stages of stellar evolution of stars between 1 and $7 M_{\odot}$. Most likely, they result from the explosion of a C/O WD with a mass close to the Chandrasekhar limit $\left(M_{C h}\right)$, which accretes matter through Roche-lobe overflow in a single degenerate scenario (SD) (Whelan \& Iben 1973), or merging of two degenerate WDs (DD) (Webbink 1984, Iben \& Tutukov 1984). We regard SDs as most likely for the majority of SNe Ia because of the homogeneity in light curves and spectra, though there is strong evidence of contributions of both to the SNe Ia population (see Hoeflich 2006, and references therein). One of the keys is the empirical relation between maximum brightness and the rate of decline, dm15 (Phillips 1993). From theory, $d m 15$ is well understood: LCs are powered by radioactive decay of ${ }^{56} \mathrm{Ni}$ (Colgate \& McKee 1969). More ${ }^{56} \mathrm{Ni}$ increases the luminosity and causes the envelopes 


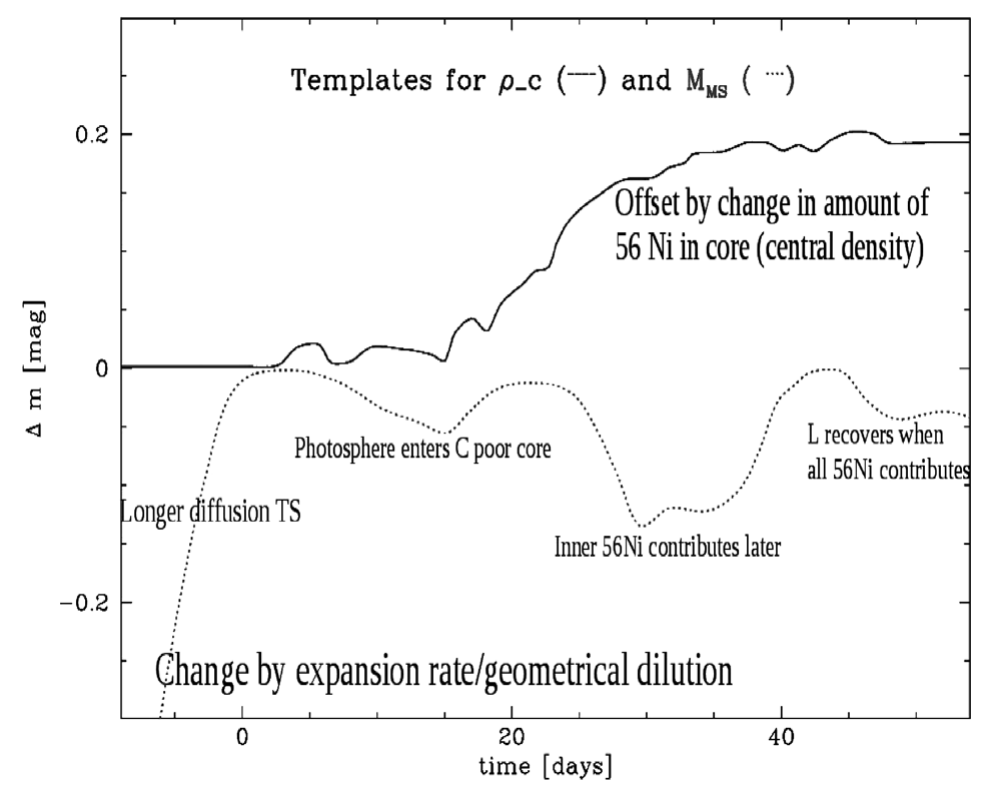

Figure 1. Principle components based on theoretical models. We show the difference in $\mathrm{V}$ brightness $\Delta m(t)$ (in magnitudes) as a function of time (in days) relative to a reference model with solar metallicity, a main-sequence mass $M_{M S}$ of $7 M_{\odot}$, and a central density of the exploding WD of $\rho_{c}=2 \times 10^{9} \mathrm{~g} \mathrm{~cm}^{-3}$. The plots are for models with $7 M_{\odot}$ and $5 \times 10^{9} \mathrm{~g} \mathrm{~cm}^{-3}$, respectively. The annotations on the graph give the main reason for the differences.

to be hotter. Higher temperature means higher opacity and, thus, longer diffusion time scales and, thus, slower decline rates after maximum light (Hoeflich et al. 1996, Nugent et al. 1997, Maeda et al. 2003, Kasen \& Woosley 2009). The existence of a dm15-relation holds up for virtually all scenarios as long as there is an excess amount of stored energy to be released (Hoeflich et al. 1996).

Within SDs, the favorite models are $M_{C h}$ explosions in which burning starts as deflagration which, at some point, transitions to a detonation (DDT) (Khokhlov 1991). The ${ }^{56} \mathrm{Ni}$ production depends mostly on the DDT, i.e. one parameter. The expanding SN-envelopes have similar velocity and density structures because they have the same masses, most of the WD undergoes burning, and the nuclear binding energies of the burning products are nearly the same. A dispersion of $0.2^{m}-0.3^{m}$ is expected (see Figure 1). Its origin can be related to the progenitor and accretion from the donor star: changes in the metallicity and the main-sequence mass, $M_{M S}$, will change the size of the explosion energies by about $20 \%$ because of their influence on the size of C-depleted core formed during stellar He-core burning (Hoeflich et al. 1998, Domínguez et al. 2001). Increasing accretion rates will decrease the central WD density $\rho_{c}$ at the time of explosion and, consequently, an increased electron capture will produce more stable iron-group elements at the expense of ${ }^{56} \mathrm{Ni}$.

High precision LCs have been obtained at LCO for 18 local SNe Ia (Contreras et al. 2010, Folatelli et al. 2010) and the individual, theoretical signatures have been recovered (Hoeflich et al. 2010). Note that a similar pattern can be produced by off-center ${ }^{56} \mathrm{Ni}$ distributions but $\Delta m$ increases later at $\approx 60$ to 100 days. 

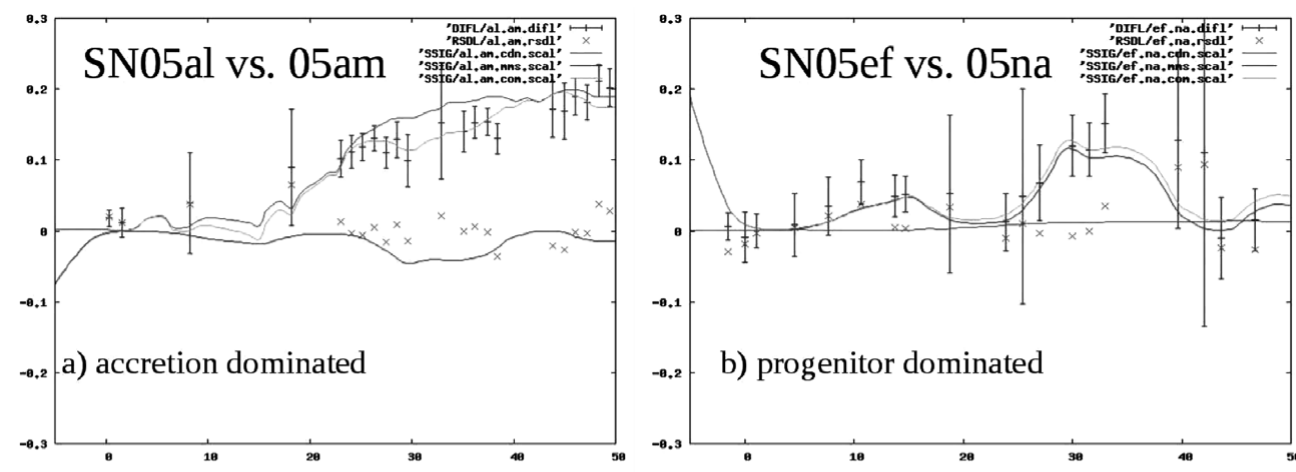

b) progenitor dominated
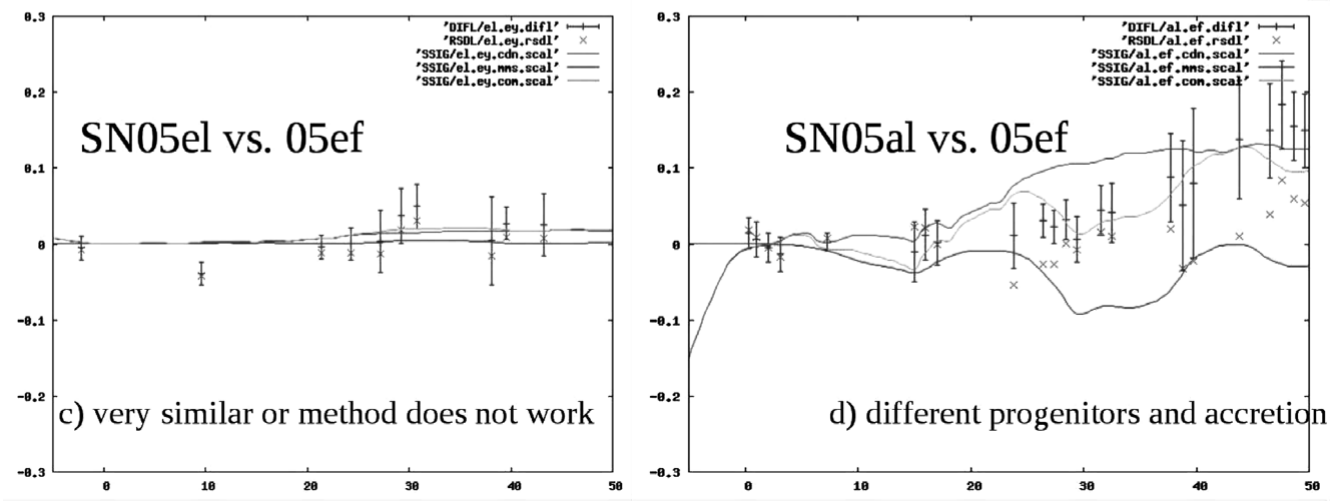

Figure 2. Best fits to the observations of individual pairs of SNe Ia. We give the weighted components, their sums along the observations (with error bars) and the residuals (crosses) for cases which are dominated by the central density/accretion rate (upper left), in the progenitors (upper right), similar $\rho_{c}$ and $M_{\odot}$ (lower left), and a mixed case (lower right). Note that the residuals are small and, within the error bars, consistent with zero.

\section{Diversity of Type Ia Supernovae}

Secondary parameters: Two SNe Ia may differ in both $\rho_{c} /$ accretion and the Cdepleted core $/ M_{M S}$. Therefore, we employed component analysis to study secondary parameters. We use $\mathrm{V}$ because this color is hardly affected by metallicity, asphericity effects, and k-corrections. The differences $\Delta m(t)$ in LC pairs are described by

$$
\Delta m_{i j, o b s}(t)=\sum_{k=1,2} \lambda_{k}(i j) f_{k}(t)+\operatorname{Res}_{i j}(t)
$$

with $\lambda_{k}(i j)$ being the coefficients for a pair of SN $i$ and $j, f_{k}(t)$ the principle components, and $\operatorname{Res}(t)$ the residuals. In our sample, we include $18 \mathrm{SNe}$ from LCO, i.e. 153 pairs. Only $36 \lambda_{i j}=g_{k}(i) / g_{k}(j)$ 's are independent where $g_{k}(i)$ is the eigenvalue of $f_{k}$ to be attributed a specific SNe Ia. By solving the overdetermined system for $g_{i}$ using a simplex method (Nelde \& Mead 1965), we obtain the most likely values for $\tilde{\lambda}_{k}(i j)$. In Figure 2, the pairs SN2005al/SN2005am, SN2005el/sn2005ef, SN 2005ef/SN 2005na and SN 2005al/SN2005ef are given for $\tilde{\lambda}_{k}(i j)$. Overall, the residuals are consistent with zero, but the fits are not unique due to errors in brightness and time coverage (see Figure 3). Remapping the individual $g_{k}(i)$ to $M_{M S}$ and $\rho_{c}$, shows that (a) values of $\rho_{c}$ are evenly distributed from $1 \times 10^{9} \mathrm{~g} \mathrm{~cm}^{-3}$ to $7 \times 10^{9} \mathrm{~g} \mathrm{~cm}^{-3}$, i.e. densities close to producing an accretion induced collapse (AIC), (b) SNe Ia come from massive progenitors with 

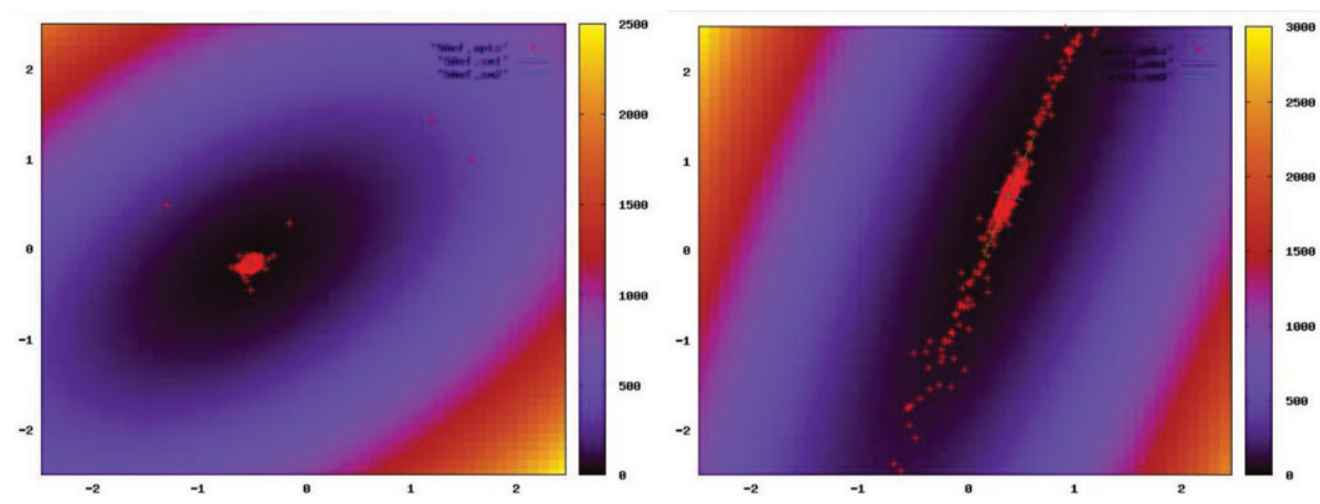

Figure 3. Probability distributions of $\tilde{\lambda}_{i j}$ for the pairs of SN2005al/SN2005am and SN2005el/sn2005ef based on MC solutions for the overdetermined system. Sparse time coverage produce large uncertainties in the eigenvalues.
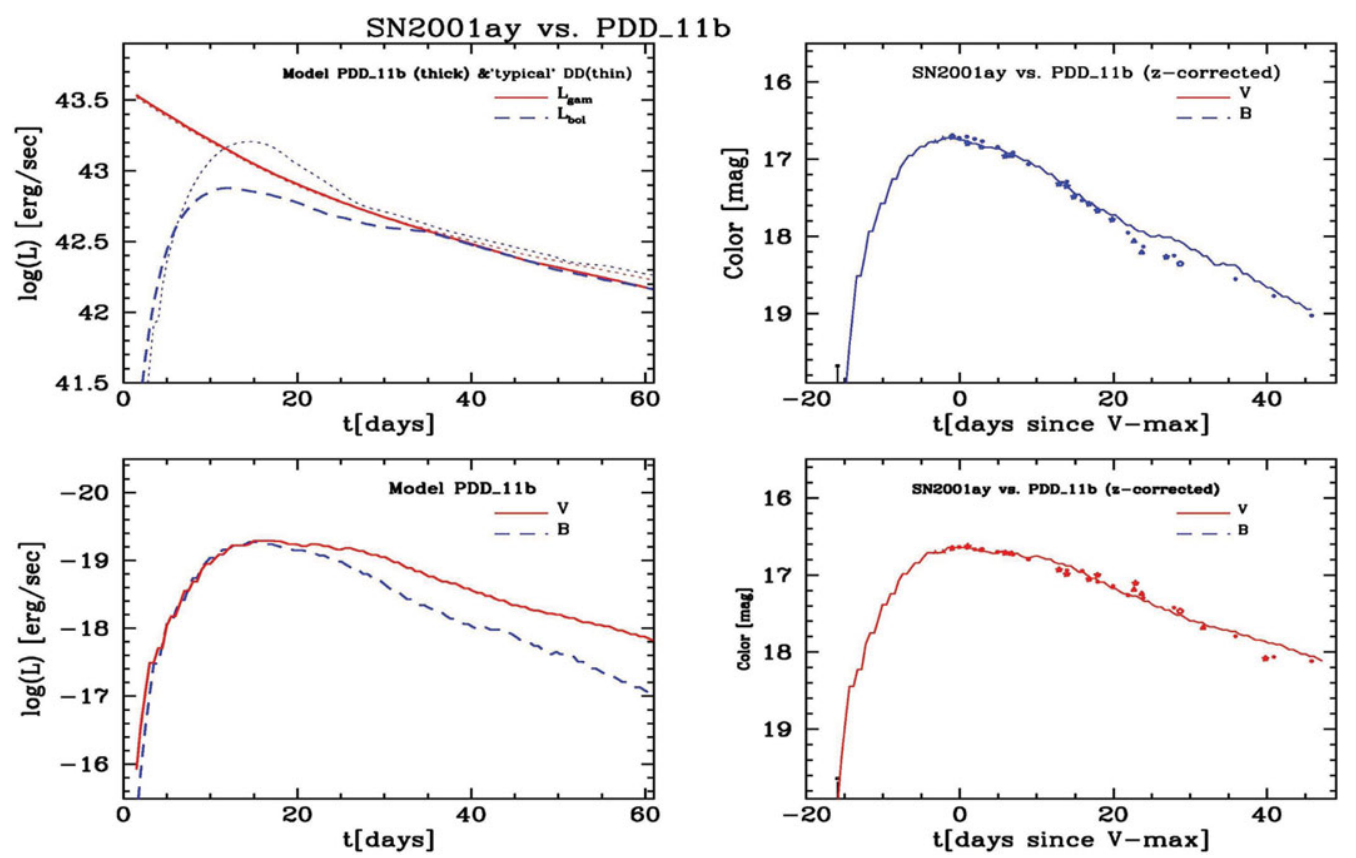

Figure 4. B and V LCs of SN2001ay (Krisciunas et al. 2011) in comparison with theory. We give the instantaneous deposition by radioactive matter for PDD11b, a pulsating model with a carbon rich core, and a classical DD model (upper left), and the B and V LCs (lower left). The DD model has been scaled by 0.037 dex to equal the gamma-ray input at maximum light. The comparison between SN2001ay and PDD11b in B (upper right) and V (lower right) as a function of time since maximum light in $\mathrm{V}$ observed. We assume a distance modulus $\mathrm{m}-\mathrm{M}$ of $35.75^{m}$, and a total reddening $\mathrm{E}(\mathrm{B}-\mathrm{V})$ of $0.06^{m}$.

$M \geqslant 3 M_{\odot}$ but the two subluminous SNe Ia indicate $M_{M S}$ between 1-2 $M_{\odot}$. All pairs of SN1991t-like objects show $\lambda_{k}(i j)=0$ in all components. Either they are very similar despite differences in $d m 15$, or they lack a central region of high densities and similar $\mathrm{C} / \mathrm{O}$ in the progenitors as may be expected for mergers (DD) or pulsating delayed detonation models. For more details and a complete analysis of all 28 LCO SNe Ia, see Sadler et al. (in preparation). 
SN2001ay shows that nature is even more diverse. SN2001ay is slower than any SNe Ia known, and has a fast rise of some 16 days (Krisciuanas et al. 2011). SN2001ay would be brighter by about $1^{m}$ based on $d m 15$ and the distance to the host galaxy. In fact, $d m 15$ is slower than implied by the instantaneous energy input by radioactive decays $\dot{E}_{\gamma}$. We submit that, still, this SN can be understood within the physics underlying the $d m 15$ relation, and in the framework of pulsating delayed detonation models originating from a $M_{C h}$ mass WD but with a core of some $80 \% \mathrm{C}$ rather instead of the $15-20 \%$ usual for stellar central He burning. Higher $\mathrm{C}$ fraction means more nuclear energy from ${ }^{12} \mathrm{C}(\alpha, \gamma){ }^{16} \mathrm{O}$ by $\approx 40 \%$ and faster expansion of the inner layers. Faster expansion means that a larger fraction of the energy by ${ }^{56} \mathrm{Ni}$ decay goes into expansion work rather than boosting the luminosity at maximum light, lower optical depth, and shorter rise time. In our models, the maximum brightness is smaller than the instant radioactive energy release $\dot{E} \gamma$ (Arnett 1988), and the light curves approaches $\dot{E} \gamma$ "from below". Our model agrees reasonably well with the observations (Figure 4). We can only speculate about the reason for a high $\mathrm{C}$ abundance. During the early stages of central He burning, high $\mathrm{C}$ abundances are produced by ${ }^{4} \mathrm{He}(2 \alpha, \gamma){ }^{12} \mathrm{C}$ burning but, at the end, ${ }^{12} \mathrm{C}(\alpha, \gamma){ }^{16} \mathrm{O}$ depletes ${ }^{12} \mathrm{C}$ unless strong mixing of He avoids this phase. A possible path may be a common envelope like in the progenitor of SN1987A. For more details, see Baron et al. (in preparation).

\section{References}

Arnett W. D. 1988, ApJ 331, 377

Colgate, S. A. \& McKee, C. 1969, ApJ, 157, 623

Contreras, C., et al. 2010, AJ, 139, 519

Domínguez, I., Höflich, P., \& Straniero, O. 2001, Nuclear Physics A, 688, 21

Folatelli, G., et al. 2010, AJ, 139, 120

Hoeflich, P., et al. 2010, ApJ, 710, 444

Hoeflich, P. 2006, NuPhA 777, 579

Hoeflich, P., Wheeler, J. C., \& Thielemann, F. K. 1998, ApJ, 495, 617

Hoeflich P., Khokhlov A., Wheeler C., Phillips M. M., \& Suntzeff N. B. 1996, ApJL, 472, L81

Hoyle, F. \& Fowler, W. A. 1960, ApJ, 132, 565

Iben, I., Jr. \& Tutukov, A. V. 1984, ApJS, 54, 335

Kasen, D. \& Woosley, S. E. 2009, ApJ, 703, 2205

Khokhlov, A. 1991, A\& A 245, 114

Krisciunas, K., et al. 2011, AJ, 142, 74

Maeda, K., Mazzali, P., Deng, J., et al. 2003, ApJ, 593, 22

Nelder J. A., Mead R., 1965, CJ 7, 308

Nugent, P., Baron, E., Branch, D., Fisher, A., \& Hauschildt, P. H. 1997, ApJ, 485, 812

Phillips, M. M., 1993, ApJ, 413, 105

Webbink, R. F. 1984, ApJ, 277, 355

Whelan, J. \& Iben, I., Jr. 1973, ApJ, 186, 1007 